\title{
An Integrated ERP with Web Portal
}

\author{
Yehia M. Helmy ${ }^{1}$, Mohamed I. Marie ${ }^{2}$, Sara M. Mosaad ${ }^{3}$
}

(1) Managment Information System Department, Faculty of Commerce \& Business administration, Helwan University yehiahelmyeyahoo. com

(2) Information System Department, Faculty of Computers and Information, Helwan University mohamedmarie71@gmail.com

(3) Managment Information System Department, Faculty of Commerce \& Business Administration, Helwan University

\author{
salwi870hotmail.com
}

\begin{abstract}
Making the right decision at the right time requires that the data should be available at internet time not after the event when it is too late to do anything. So the need for a web portal has been increased in order to support decision making in the different organizational levels at internet time by providing features such as, customization, personalization, support for collaboration and notification that support managers in making the right decision at internet time. All this can be achieved by applying the (BI) tools on "One version" data store that contains data from legacy ERP system after applying the ETL tools over it. Also the Data marts will be used in order to shorten the response time of queries generated by the users.
\end{abstract}

\section{Keywords:}

Business Intelligence, ETL, Enterprise Resource Planning, ERP, Web portal, legacy ERP System, One version data store

\section{1- Introduction}

The huge amount of data in ERP systems may lead to an increase of responding time for the managers' queries. The web portal will provide representative and useful information for managers at different organizational levels to assist them in taking decisions and to help and support them in their activities such as analyzing departmental data, planning and forecasting activities for their decision area. That's why we should consider applying an ERP portal in order to improve the Enterprise's performance.

The ERP portal enables managers at every departmental level to have a customized view that extracts information from transactional sources and summarizes it into meaningful indicators in short time.

Data analysis, reporting, and query tools can help business users through a sea of data to synthesize valuable information from it - today these tools collectively fall into a category called 'Business Intelligence'[1]

ERP system appears to be the first system to be applied to manage the construction of the supply chain. Its purpose is to integrate all departmental and functional information that flow across the company onto a single computer system that fulfill all the management needs of the enterprise. With the installation of additional features in the latest version of the ERP software, external suppliers and customers are taken as part of the chain that the system manages. The

DOI : 10.5121/acij.2012.3501 
research by Gattiker proves that ERP provides many benefits such as increasing efficiency, quality, productivity and profitability.[2]

ERP systems are implemented in many organizations for operational and transactional processing such as: financials, inventory, purchase, order management, production. Managers at operational levels of the organization require detailed reports with daily operational activities, while the managers at strategic and tactical levels of the organization often requires reports with aggregated data from ERP and non-ERP application sources for making strategic and tactical decisions. The usual reports developed from daily transactions do not satisfy the business needs, an executive cannot take a real time decision. [3].

BI refers to using of the technology to collect and effectively use information in order to improve the business potency. An ideal BI system enables the organization's employees, partners, and suppliers to easily access, analyze and share the information they need to effectively do their jobs. BI provides critical insight that helps organizations' managers make informed decisions.

BI facilitates scrutinizing every aspect of business operations to find new revenue or minimizing additional cost savings by supplying decision support information.[4]

\section{LEGACY ERP SYSTEMS CHALLENGES}

Legacy ERP system challenges can be summarized as[5]:

- $\quad$ Organizational resistance to substituting it with new one.

- $\quad$ Lake of support or maintenance.

- $\quad$ No upgrading provided.

- $\quad$ Non-robust and incomplete ERP packages.

- $\quad$ Complex and undefined system interfaces.

- $\quad$ Middleware technology bugs.

- $\quad$ Poor custom code.

- $\quad$ Poor system performance.

- $\quad$ Lack of information needed for making good decision.

- $\quad$ Loss of employee morale as they suffer with screens and processes that make their jobs harder, not easier.

- Increased costs due to maintenance expenses and the high-cost associated with customizations needed to adapt the system to business needs.

- $\quad$ The proposed model provides an adequate solution to most of these challenges as shown in the following section.

\section{2- THE PROPOSED MODEL}

The proposed model were built over an existing legacy ERP systems which composed of several modules (accounting, sales, inventory... etc) from different vendors and because of the delay in responding the queries and the resistance for changing the existing legacy ERP systems, so the (BI) tools were the best solution to all this challenges. Also ETL tools were applied to perform the following processes over the legacy ERP:[6]

- Extract data from a source application's database or file system

- perform necessary data transformations such as correcting rudimentary data errors, summarizing data, converting and unifying data codes (e.g., source Terminology and application \#1 uses "M" for male and "F" for female, while source application \#2 uses an integer of 1 for male and 2 for female; these would typically be converted to some common code in the data warehouse as data is consolidated)

- Load the transformed and "cleansed" data content into the data warehouse "OneVersion Data Store". 
ETL tools supply the data warehouse with the suitable formatted data. A dedicated data warehouse build especially for ERP is used for this model. A several data marts are extracted from the Data warehouse to facilitate good interactive query response. Finally according to the need of customization, personalization, support for collaboration and notification, a web portal has been used for the representation of the output of the (BI).

As shown in Fig 1 which illustrates the components of the proposed model which are:

1. Legacy ERP System.

2. ETL.

3. Data Warehouse "One-Version Data Store".

4. Data Marts.

5. Web Portal.

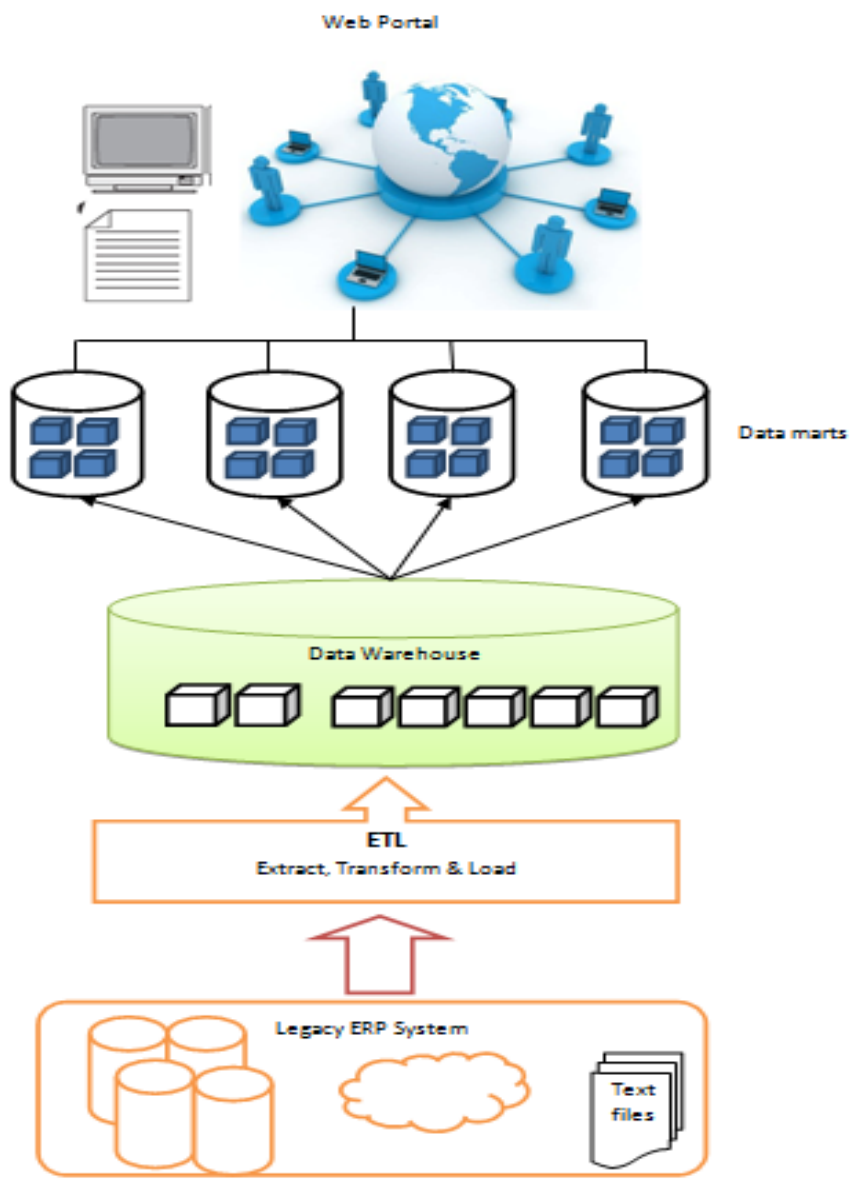

Fig 1: The Proposed Model

\section{4-1 Model components}

\section{4-1-1 Legacy ERP systems}

Is an old computer system that still in use despite the availability of more efficient processes or technologies. A legacy ERP system refers to computer related methods that can be used in relation to other outdated tools, methods or human behavior. The disadvantages of legacy ERP systems are that it may refer to terms or procedures which are no longer related in the current context of the system and can therefore lead to confusion. Also integrating a new system with a legacy system is very difficult due to software incompatibility. The causes of retention is that a legacy system may be kept in service due to the high cost involved in replacing or redesigning 
the system, also a legacy system may also require constant availability such as air traffic control system which prohibit easily taking the legacy system out of service to redesign up to date system. [7]

\section{4-1-2 ETL}

Extraction-Transformation-Loading (ETL) tools are pieces of software responsible for the extraction of data from several sources, their cleansing, customization and insertion into a data warehouse.

In Fig 2, describes generally ETL processes. The data stores that are involved in the overall process which is the legacy ERP systems are placed in the bottom layer of the figure, the original data providers are placed on the left side. As shown in the upper left part of figure the data from these sources are extracted by extraction methods, which provide either complete snapshots or differentials of the data sources.

Then, these data are propagated to the Data Staging Area (DSA), at this stage they are transformed and cleaned them they are loaded to the data warehouse. The data warehouse is placed in the right part of Fig 2 and comprises the target data stores, i.e., fact tables and dimension tables. Eventually, the loading of the transformed and cleaned data to the central warehouse is performed through the loading activities placed on the upper right part of the figure.[8]

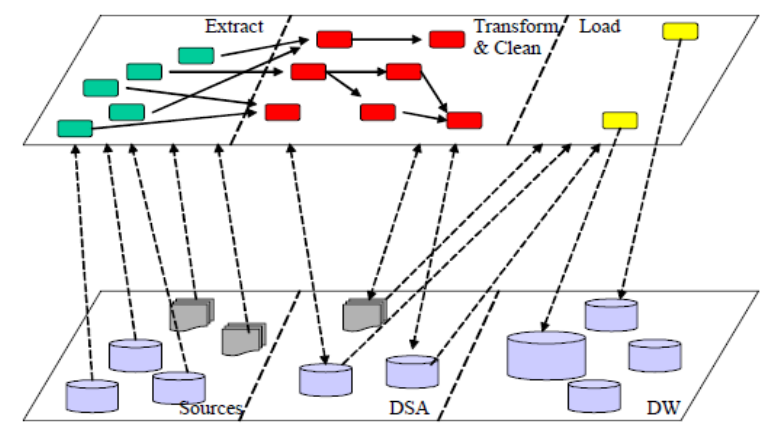

Fig 2: The environment of ETL processes[9]

\section{4-1-3 Data Warehouse "One Version Data Store"}

Is the systems that contain operational data - the data that runs the daily transactions of the enterprise business-contain information that is useful to business analysts. For example, analysts can use information about which products were sold in which regions at which season in order to project future sales. However, there are several problems if analysts access the operational data directly:

- They might not have the expertise to query the operational database.

- Performance is essential for many operational databases, such as databases for a bank, so that the system cannot handle users that make ad-hoc queries.

- Usually the operational data is not in the best format for use by business analysts.

Data warehousing solves these problems. In data warehousing, creating stores of informational data that is extracted from the operational data and then transformed for end-user in order to make decision. For example, a data warehousing tool might copy all the sales data from the operational database by performing calculations on the operational data and then summarize the data, and write the summarized data to a separate database from the operational data. End-users can query the separate Data Warehouse without impacting the operational databases [10]. Data Warehouse is used as it is a subject-oriented which provides information about a particular subject instead of about a company's ongoing operations, integrated as data is gathered from a 
variety of sources, time-variant as data is identified with a particular time period., and nonvolatile as more data is added but data is never removed. This enables management to gain a consistent picture of the business.[11].

According to a Panian report, 57\% of companies said their organizations were using their general corporate or enterprise data warehouses to support their ERP applications, as opposed to $43 \%$ who were using a separate data warehouse intended specifically for ERP analytics. Using a "one-version data store" Data warehouse provides a consolidated view which offers the optimum capabilities for an enterprise data analysis and reporting. The drawback to this approach is that it typically requires a considerable undertaking in which redesigning the enterprise data warehouse to incorporate ERP models and reporting processes is essential. On the other hand, using a dedicated data warehouse built specifically for ERP analysis will allow faster application development and enables realization of benefits quickly from the ERP initiative. However, it will not offer the consolidated enterprise analysis and reporting capabilities provided by the enterprise data warehouse [12]. So, in the proposed model we use a dedicated data warehouse which is built specially for ERP.

Data Warehouse is a single, complete and consistent(reliable, dependable) store of data obtained from a variety of different sources made available to end users can understand and use in a business context The data stored in the warehouse is uploaded from the operational systems. The data may pass through an operational data store for additional operations before it is used in the DW for reporting. A data warehouse maintains its functions in three layers: staging, integration, and access. Staging layer is used to store raw data for the developers. The integration layer is used to integrate data and to have a level of abstraction from users. Finally the access layer is used for getting data out from the repositories to the users.[13]

The purpose of a data Warehouse in our model is to establish a comprehensive source of data from legacy ERP systems.

\section{4-1-4 Data Marts}

Data marts are smaller, subject oriented structures which are extracted from a Data Warehouse. Data marts are suitable for environments where there are many sources for the data, data can be classified into single subject based repositories and data needs to be pre-summarized as volumes are large in order to facilitate good response for the queries and to avoid resource contention with other system users.

Although data marts are not usually normalized structures Entity Relationship (ER) modeling can still be useful in data mart design. Starting with producing the conceptual model which shows the entities in a detailed way and the relationships between them. The logical model adds the significant attributes and key information and the physical model provide the attribute's characteristics and its physical naming [14].

\section{4-1-5 Web Portal}

Because ERP systems are implemented in many organizations for operational and transactional processing such as: financials, inventory, purchase, order management. In organizations the operational managers require detailed reports for making operational decisions, on the other hand strategic and tactical managers require reports with aggregated data from ERP application sources for strategic and tactical decision as the usual reports developed from daily transactions does not satisfy the business needs[15]. So The Web portal is used as a means of communication and as a decision support system on high-end graphical user interfaces. The main advantage of implementing an ERP web portal is to combine as many functionalities as possible into a single, integrated web portal that runs on a "one-version data store", in order that the various departments can easily share information and communicate with each other at internet time[16]. Web portal may not be the only way to make data available at internet time but it also provides collaboration and information sharing at the extended enterprise level which makes the Web portal solution very attractive. 


\section{Web portal architecture}

As shown in fig 3 the Web Portal architecture consists of the following three layers:

A. The Presentation or user Layer.

B. The Infrastructure Layer.

C. The Information and Application Layer.

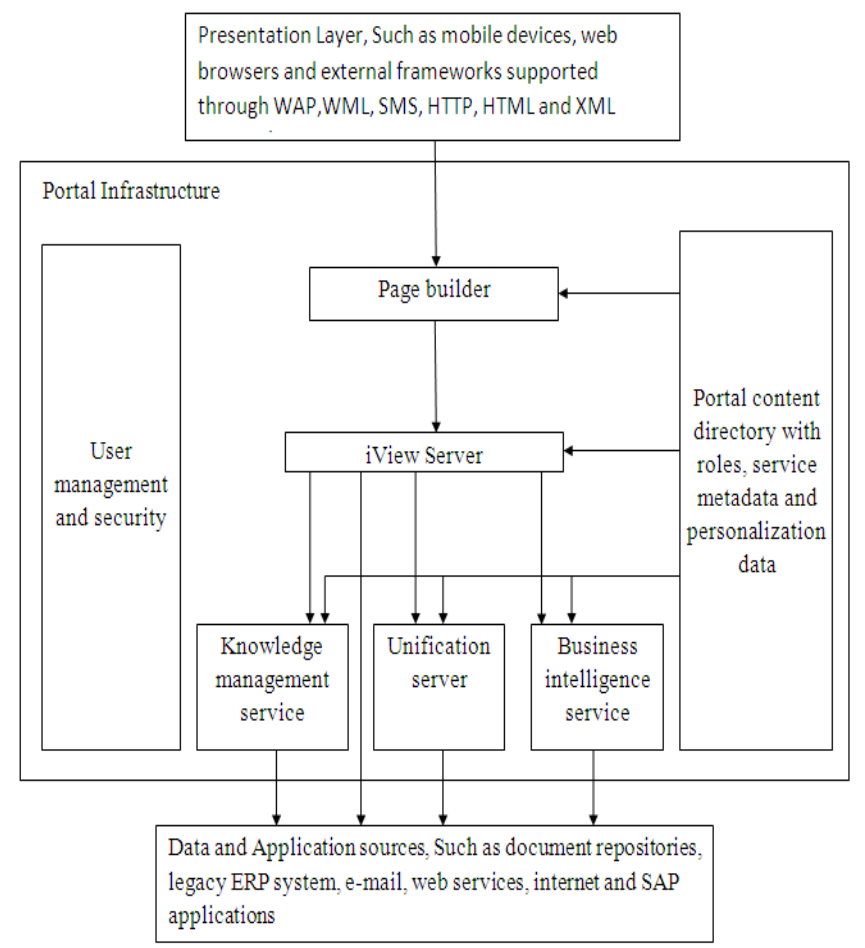

Fig 3: Portal Infrastructure[17]

\section{A. The Presentation or User Layer}

Is where the end-users interact with the portal through requests for information and application functionality in some underlying systems. The users can be employees, customers, suppliers, or partners of the firm. Which information or applications a user is able to interact with is governed by the user's "role(s)." A role defines an activity set that a portal user, internal or external person or application, undertakes in order to achieve a desired business objective. Roles, rather than persons or functional position, define how a work-task should be performed. Each role can only access information and applications that are included in that specific role. For example, a "sales assistant" can only access the applications and information associated with this role. Finally, End-users may use a variety of devices, such as mobile phones, pocket PCs, and laptops.

\section{B. The Infrastructure Layer}

It functions as a broker between the presentation layer and the information and application layers. It consists of:

- Page builder, used to design user interfaces.

- User management and security, the administrative part of the portal, where user data repositories are stored, and it is based on LDAP (Lightweight Directory Access Protocol).

- Portal content directory,

- iView Server, functions as a connector to the different information and applications 
sources. It has a repository of predefined connectors, but a firm may develop new connectors. Connectors can be built into most programming languages and will give a consistent XML-based output.

- Knowledge management service manages the unstructured data and information and functions as a content management system.

- Unification server manages the integration of data and information from disparate systems.

- Business intelligence service contains analysis and reporting, OLAP Service manages functionality, and data warehouse capabilities. the Unification Server.

\section{The Information and Application Layer}

The information and application layer consists of different sources of data and information and the different applications a user may need to fulfil his or her work-tasks, i.e., "one-version data store".

The ERP web portal has the following features:

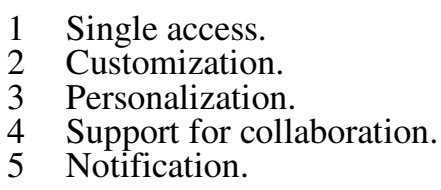

1- Single access and authorization point means access, through one access point (i.e., the portal), to the following:

- All internal and external applications, e.g., ERP system, CRM system, e-commerce tools, SCM system, e-mail, calendar, and legacy systems

- Information, e.g., company newsletters, financial statements, product shipments, document repositories.[17]

2- Customization means provide content tailored to meet the needs of the portal visitor. In fact, the power of the web portal lies, to a large extent, in its ability not only to consolidate information but also to provide that information in a specialized manner.[8]

3- Personalization means adapting the portal to individual needs by adding or deleting functionality.

4- Support for collaboration, by providing a dynamic and flexible way for customers, suppliers, partners and employees from different companies to be active participants in the decision making process. This collaboration goes far beyond simply sharing information through a Web browser[18].

5- Notification services provide users with the needed information any time, empowering them to make timely and informed decisions. Notifications will be sent to users' mobile devices as SMS (Short Message Service) or to their email accounts[3].

\section{6- Conclusions}

Using a web portal based on (BI) tools gives an integrated, web-based solution that enables legacy ERP's partners to create and share relevant information, based on data marts which are derived from a "one-version data store". Customers and outsourcing vendors may demand access to information provided to internal ERP users - such as order status, inventory levels, and invoice data, so having ERP web portal enables seamless data access to the authenticated users at the right time from everywhere. Also the web portal facilitates the customization, personalization, support for collaboration and notification with a single access to different reports with multiple styles and formats and with different extensions. All This enables ERP partners to make global, faster, and more informed decisions, to gain a more holistic and accurate view of business, without rebuilding a new ERP and substitutes the legacy running 
one.

\section{References}

[1] T. A. Horakh, H. Baars, and H. G. Kemper, "Mastering Business Intelligence Complexity-A Service-Based Approach as a Prerequisite for BI Governance," 2008.

[2] R. Deraman, H. Salleh, A. M. Beksin, A. M. Alashwal, and B. Chafe, "The roles of information and communication technology (ICT) systems in construction supply chain management and barriers to their implementation," African Journal of Business Management, vol. 6, pp. 24032411, 2012.

[3] M. Chen, "Providing web services to mobile users: the architecture design of an m-service portal

" 2005.

[4] S. N. S. G R Gangadharan, "Business Intelligence Systems: Design and Implementation Strategies," 2004.

[5] H. Krasner, "Ensuring e-business success by learning from ERP failures," IT professional, vol. 2, pp. 22-27, 2000.

[6] S. S. Alan Simon, "Data Warehousing and Business Intelligence for e-Commerce," 2008.

[7] R. C. Seacord, D. Plakosh, and G. A. Lewis, Modernizing legacy systems: software technologies, engineering processes, and business practices: Addison-Wesley Professional, 2003.

[8] D. B. G. Jon P. Gant, "Web portal functionality and State government E-service," 2002.

[9] T. M. Simatupang and R. Sridharan, "The collaborative supply chain," International Journal of Logistics Management, The, vol. 13, pp. 15-30, 2002.

[10] S. Negash, "Business intelligence," Communications of the Association for Information Systems, vol. 13, pp. 177-195, 2004.

[11] W. H. Inmon, Building the data warehouse: Wiley-India, 2005.

[12] Z. Panian, "Supply chain intelligence in e-business environment," WSEAS Transactions on Information Science and Applications, vol. 2, pp. 1079-1084, 2005.

[13] M. F. Khalil, H. Acharya, and M. Alam, "Data Mining and warehousing for Comprehensive Web Based Project Management Software," 2012.

[14] P. O’Hara, "Integrating Business Intelligence with ERP To Improve Operational and Strategic Planning Information in Small and Medium-sized Enterprises

" 2009.

[15] I. L. ADELA BÂRA, MANOLE VELICANU, VLAD DIACONIȚA, IULIANA BOTHA, "IMPROVING QUERY PERFORMANCE IN VIRTUAL DATA

WAREHOUSES," 2008.

[16] C. Tarantilis, C. Kiranoudis, and N. Theodorakopoulos, "A Web-based ERP system for business services and supply chain management: Application to real-world process scheduling," European Journal of Operational Research, vol. 187, pp. 1310-1326, 2008.

[17] F. Adam and D. Sammon, The enterprise resource planning decade: lessons learned and issues for the future: IGI Global, 2004.

[18] B. R. N. Stefanovica, D. Stefanovicc " Supply chain intelligence " 2007. 